\title{
An atomic force microcopy study of the mechanical and electrical properties of monolayer films of molecules with aromatic end groups
}

\author{
Liang Fang ${ }^{1}$, J. Y. Park ${ }^{1}$, H. Ma ${ }^{2}$ and A. K.-Y. Jen ${ }^{2}$, and M. Salmeron ${ }^{1, a}$ \\ ${ }^{1}$ Molecular Foundry, Materials Science Division, Lawrence Berkeley National Laboratory, \\ Berkeley, California 94720 \\ ${ }^{2}$ Department of Materials Science and Engineering, University of Washington, Seattle, \\ Washington 98195
}

The effect of intermolecular $\pi-\pi$ stacking on the electrical and mechanical properties of monolayer films molecules containing aromatic groups was studied using atomic force microscopy. Two types of aromatic molecules, (4-mercaptophenyl) anthrylacetylene (MPAA) and (4-mercaptophenyl)-phenylacetylene (MPPA) were used as model systems with different $\pi$ - $\pi$ stacking strength. Monolayer films of these molecules on Au(111) surfaces exhibited conductivities differing by more than one order of magnitude, MPAA being the most conductive and MPPA the least conductive. The response to compressive loads by the AFM tip was also found to be very different for both molecules. In MPAA films distinct molecular conductivity changes are observed upon mechanical perturbation. This effect however was not observed on the MPPA film, where intermolecular $\pi$ - $\pi$ interactions are likely weaker.

\footnotetext{
${ }^{a)}$ Author to whom correspondence should be addressed. Electronic mail: mbsalmeron@lbl.gov
} 


\section{Introduction}

Molecular electronics is an emerging field that is drawing considerable attention due to the potential applications offered by inexpensive plastic electronics devices based on organic/polymeric materials instead of traditional silicon based solid-state semiconductors ${ }^{1-10}$.
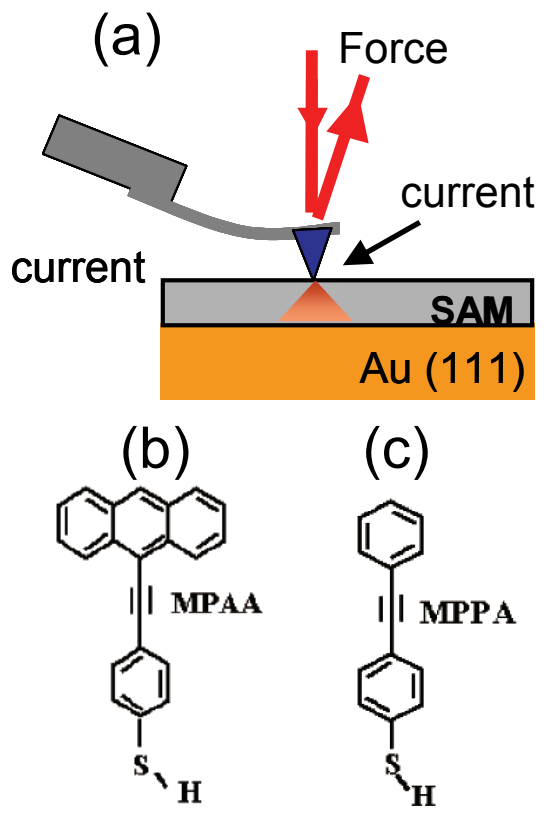

Figure 1. (a) schematic of AFM measurement of current and friction on self-assembled monolayer organic molecules / $\mathrm{Au}$ (111) surface. (b) schematic illustration of MPAA, and (c) MPPA.
Molecules with good conductive properties contain groups with their highest occupied molecular orbital (HOMO) and lowest unoccupied molecular orbital (LUMO) levels separated by only a small energy gap. Among these, double and triple $\mathrm{C}$ bonds and aromatic rings are widely used. However, semiconductors made of $\pi$-conjugated molecules suffer from intrinsically low charge carrier mobility. Unlike single crystal semiconductor materials, electrons or holes "hop" between molecules in order to carry charges ${ }^{7,8}$. Among the factors that control the "hopping" rate, intermolecular $\pi$ - $\pi$ stacking is one of the most important ${ }^{4,7,9,10}$.

Although many conjugated molecules form $\pi-\pi$ stacked molecular superstructures, phenylacetylene compounds are promising due to their stability in air and against ultraviolet light, rigid framework and the fact that they can be attached to gold strongly ( $40 \mathrm{kcal} / \mathrm{mol})$ via thiolterminated groups. Previous studies have shown that controlled self-assemblies of $\pi$-conjugated molecules can result in a 2-D superlattice on $\mathrm{Au}(111)$ surfaces at room temperature ${ }^{11}$. Here we study the electric transport properties of two such systems and the effects of mechanical perturbations.

Two conjugated molecules were studied: (4-mercaptophenyl)-phenylacetylene (MPPA) ${ }^{12-}$ ${ }^{15}$ and (4-mercaptophenyl) anthrylacetylene (MPAA) ${ }^{11}{ }^{16}$. The presence of three fused aromatic rings in MPAA is expected to result in a stronger $\pi-\pi$ intermolecular stacking than in the case of MPPA that contains one benzene ring only ${ }^{11}$. By applying mechanical load to self-assembled films of these molecules we intend to intentionally disturb the $\pi$ - $\pi$ intermolecular stacking and to 
study its effect on the electrical conductivity. This should allow us to obtain a better understanding of the influence of the $\pi-\pi$ intermolecular stacking on the electronic properties of self-assembled monolayers in general.

A related question is the effect of $\pi$ - $\pi$ interactions on the mechanical properties of such films, in particular friction. Previous studies of molecular scale friction have been focused on long-chain alkylthiol and alkylsilane molecules forming self-assembled monolayers on gold, silicon and mica substrates ${ }^{17,18}$. The friction force was found to decrease with increasing chain length and to remain almost constant beyond a certain length (approximately 10 carbons). It was proposed that the poorer packing of the shorter molecules makes possible the excitation of more energy dissipation modes, such as chain bending and tilting, rotations, gauche defects, etc., which are sterically quenched in the densely packed films formed by the longer chain molecules. For $\pi$ conjugated molecules a relationship between molecular structure and friction has not yet been established ${ }^{19,20}$. This study therefore is also an attempt to fill this hole in our understanding of the friction properties at a molecular level.

\section{Experimental}

The gold substrates were prepared by annealing a $250 \mathrm{~nm}$ thin film deposited on the borosilicate glass in a hydrogen flame to produce contaminant-free, flat (111)-oriented terraces. Solutions of MPAA and MPPA $(0.05 \mathrm{mM})$ were prepared in the following way. Ester derivatives of the molecules were first dissolved in the ethanol, then $\mathrm{NH}_{4} \mathrm{OH}$ was added to convert the -S$(\mathrm{C}=\mathrm{O})-\mathrm{CH}_{3}$ functional group into $-\mathrm{S}-\mathrm{H}$ (thiol). The gold samples were immersed in the solution for 48 hours and after removal rinsed successively with ethanol (3 min), DMF (5 $\mathrm{min})$, and ethanol ( $5 \mathrm{~min}$ ). After this they were dried by blowing dry $\mathrm{N}_{2}$ gas.

The friction experiments were performed with a commercial RHK-Technology STM/AFM system in a chamber with a base pressure of $1.0 \times 10^{-10} \operatorname{torr}^{21,22}$. All the measurements were performed at pressures below $1.0 \times 10^{-9}$. Cantilevers with spring constant of $2.5 \mathrm{~N} / \mathrm{m}$, coated with approximately $30 \mathrm{~nm}$ of conductive $\mathrm{TiN}^{23}$, were used. The normal force exerted by the cantilever was maintained constant during imaging while current and friction force between tip and sample were recorded simultaneously. The radii of the metal-coated tips were typically $30-50 \mathrm{~nm}$ before contact, as measured by scanning electron microscopy. When measured after a contact experiment however, the radii were typically found to be $80-120 \mathrm{~nm}$. The loads used in this study were small enough that no damage to the surface could be detected by inspection of images with 
$0.1 \mathrm{~nm}$ depth sensitivity as well as by the reproducibility of the friction and adhesion measurements.

\section{Results}

In Fig.2a, 2b, 2c we show topographic, current and friction force images acquired simultaneously ${ }^{24}$ under an applied load of $20 \mathrm{nN}$ on a sample covered with a monolayer of MPPA. Contours corresponding to atomic steps and terraces are visible in the images. The current over the film is $20-100 \mathrm{nA}$ at a sample bias of $+0.8 \mathrm{~V}$. This current is three orders of magnitude smaller than that found on spots exposing bare gold. The friction force over the molecular film is
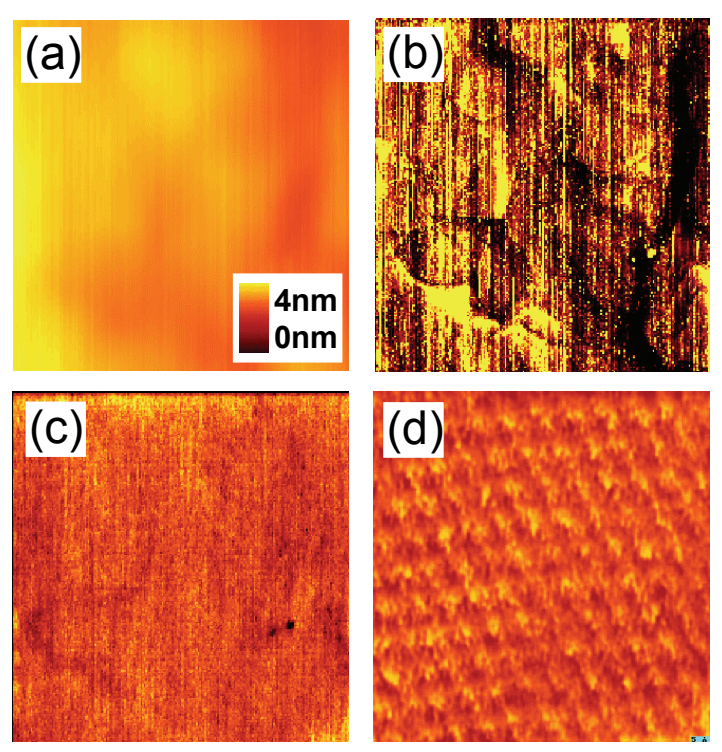

Figure 2. AFM images $(600 \mathrm{~nm} \times 600 \mathrm{~nm})$ showing the topography (a), current (b), and friction (c), on an MPPA molecular layer on Au (111). Applied load $=24 \mathrm{nN}, \mathrm{V}=+0.8 \mathrm{~V}$. (d) Lattice resolved friction image $(8 \mathrm{~nm} \times 8 \mathrm{~nm})$ of the MPPA monolayer at an external load of $0 \mathrm{nN}$. The lattice distance of self-assembled monolayer is $1.0 \pm 0.05 \mathrm{~nm}$. smaller by a factor of two than on the bare gold areas. Fig $2 \mathrm{~d}$ is a high-resolution image showing the ordered molecular structure of the film, with lattice parameter of $1.0 \pm 0.05 \mathrm{~nm}$, consistent with the STM result of Zareie et al. ${ }^{11}$. In the case of MPAA the molecular lattice could not be resolved, suggesting that the MPAA layer is more disordered than the MPPA.

Although the two molecules have the same length $(1.56 \mathrm{~nm})$, they form films of different height. Based on the STM images by Zareie et al. ${ }^{11}$ and on the van der Waals dimensions of the molecules ${ }^{25}$, a tilt angle of $12^{\circ}$ can be estimated for the MPAA molecules, corresponding to a topographic island height of $1.53 \mathrm{~nm}$. In the case of MPPA, the molecular width of $0.67 \mathrm{~nm}$ together with the lattice distance found in STM

and AFM images $(1.0 \mathrm{~nm})$, gives a tilt angle of $48 \pm 3^{\circ}$, which corresponds to an island height of $1.04 \mathrm{~nm}$, considerably lower than for MPAA.

The friction force and the current between the conductive probe and the molecular film were measured as a function of increasing load. The experiments were performed by scanning the tip in contact with the film back and forth along one line. The tip apex experiences a small displacement in the perpendicular direction due to increased bending of the cantilever with increasing load. This is a result of the approximately $15^{\circ}$ mounting angle of the cantilever relative 
to the sample $\mathrm{e}^{21,22}$. The length of the scanning track was $300 \mathrm{~nm}$ and the scan rate was 1 second per line. Figure $3 a$ and $3 b$ shows a plot of such measurements.
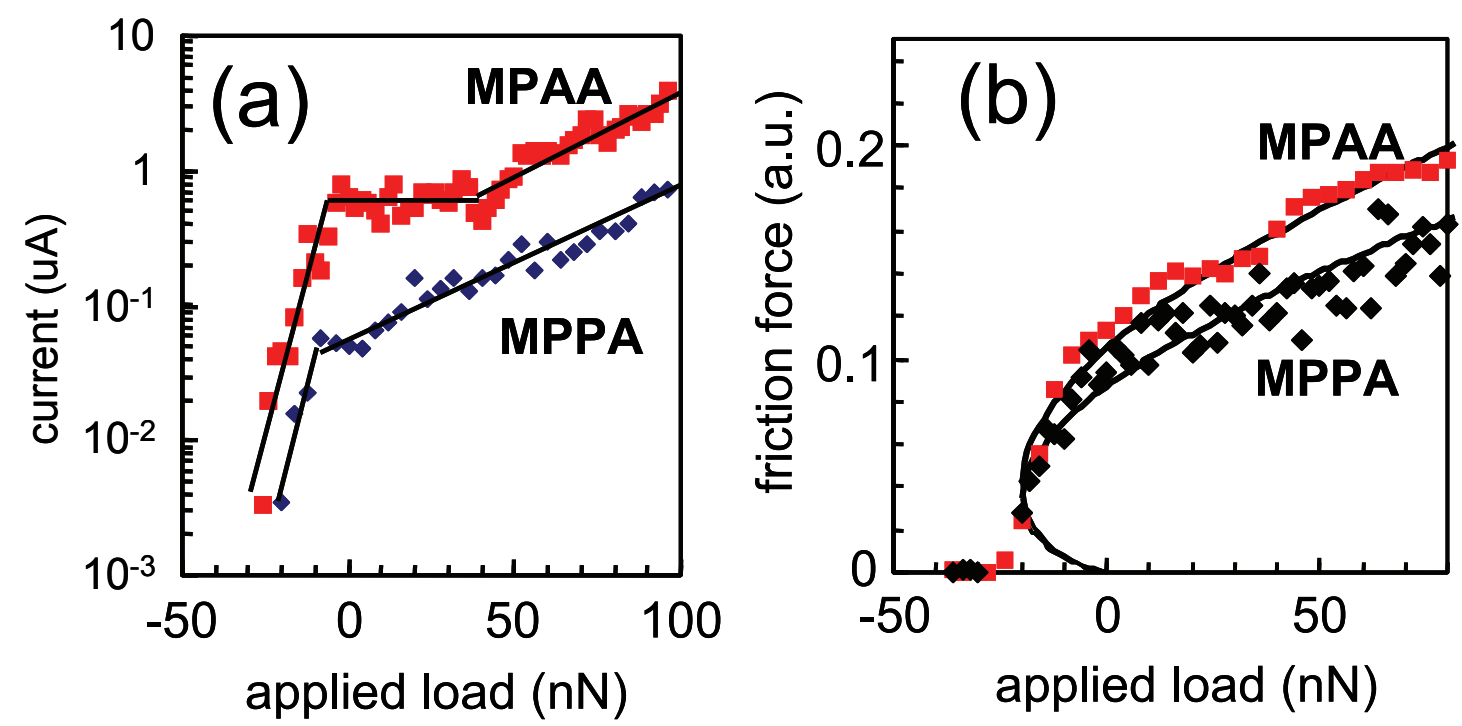

Figure 3 (a) current and (b) friction signal as a function of the applied load for MPAA and MPPA measured at the sample bias of $+1 \mathrm{~V}$. Straight lines in (a) are shown for reference. The lines in (b) are fits to the JKR elastic deformation model, assuming that friction is proportional to contact area.

We found that using the same tip, load and bias the current on MPAA was higher than that on MPPA by factor between 5 and 10. The adhesion force, determined from the pull-off force in force-distance curves, was found to be $25 \pm 2 \mathrm{nN}$ on MPPA, and $27 \pm 2 \mathrm{nN}$ on MPAA.

As shown in Fig. 3a the current increased rapidly with applied load on both MPPA and MPAA monolayers. This rapid increase, shown by the exponential Y-axis scale, cannot be explained as due to changes in contact area, because this area increases much more slowly with load $\mathrm{L}$, for example as $\mathrm{L}^{2 / 3}$ in the Hertzian model.

The different behavior of the current vs load on the two molecules is striking. First, the current through MPPA is roughly ten times smaller than that through MPAA. Second, for MPAA there is a plateau in the current extending approximately from -5 to $+40 \mathrm{nN}$ external load (or 20 to $65 \mathrm{nN}$ of total load, including the adhesion force). This plateau might indicate an increased stability or a particular stacking geometry of the MPAA molecules that is disrupted around $40 \mathrm{nN}$ ( $65 \mathrm{nN}$, including the adhesion force). Apart from this plateau, the current increases exponentially on both molecular films. In the semilog plot a high slope region is observed during initial compression, and a smaller slope region at higher loads. For both molecules however these two slopes are similar in each regime. 
The friction force, measured simultaneously, is shown in Fig. 3b. We found that, assuming proportionality between friction and contact area, both MPAA (top curve) and MPPA (lower curve) friction behavior follows well the JKR elastic deformation model, which includes adhesion forces $^{21,26-28}$.
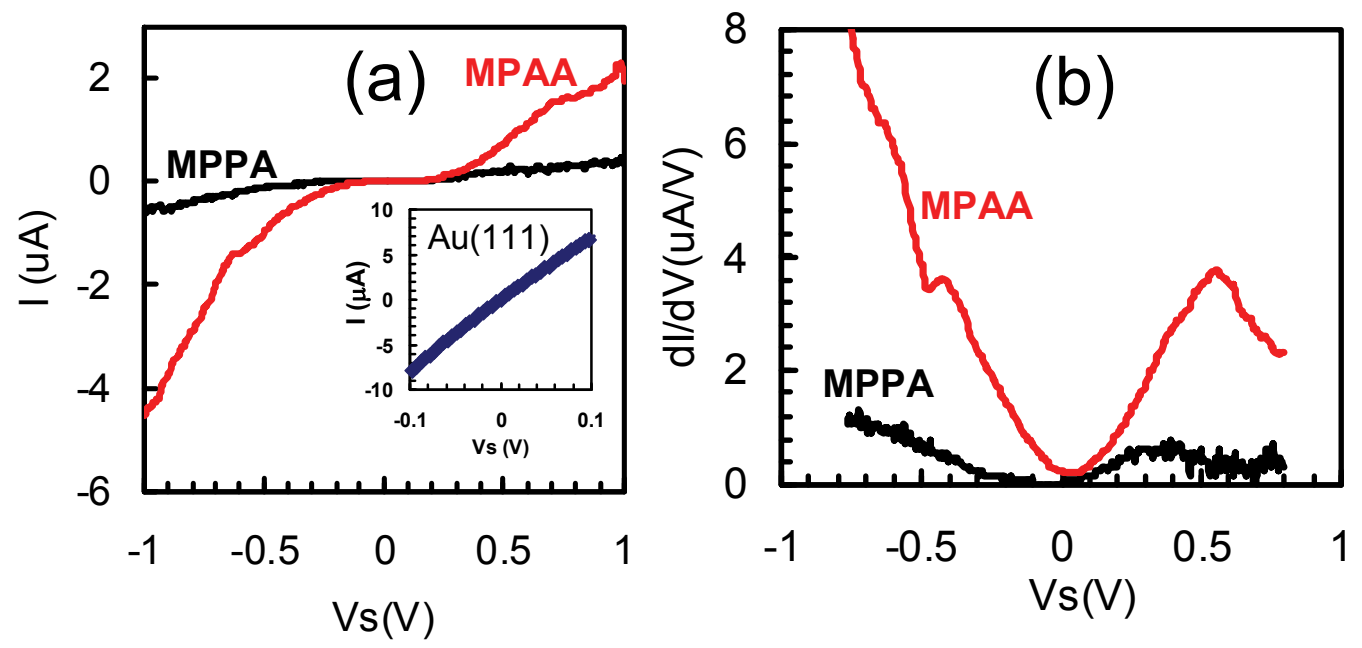

Figure 4(a) Current-voltage plot for MPPA and MPAA on $\mathrm{Au}(111)$ at the total load of $80 \mathrm{nN}$. The inset shows I-V plot measured on the bare $\mathrm{Au}$ (111) surface. (b) Conductance (dI/dV) measured as a function of the sample bias.

Figure 4 shows current (I), and conductance $(\mathrm{dI} / \mathrm{dV})$ as a function of bias at the total load (= external + adhesion) of $80 \mathrm{nN}$. The inset in Figure 4a shows the I-V curve measured on $\mathrm{Au}(111)$ surface revealing the linear behavior and higher current level expected from a metallic ohmic junction. As before, the current measured on MPAA is about ten times larger than that on MPPA. The conductance spectrum shows a resonance around $0.5 \mathrm{eV}$ corresponding to empty or LUMO states and a stronger one below $-0.8 \mathrm{eV}$ due to filled or HOMO states. Higher bias voltages could not be explored due to possible damage to the films.

\section{Discussion}

Both MPPA and MPAA molecules have benzene rings that can provide $\pi$ - $\pi$ interaction between neighboring molecules. The single benzene ring in MPPA, compared to the three fused rings of anthracene in MPAA, gives the molecule a more uniform diameter along its length, which might explain the better ordering of the MPPA film observed in the lattice resolved images. While the wider anthracene group of MPAA can provide more favorable interactions with 
neighboring molecules through increased $\pi$ - $\pi$ overlap, its less uniform molecular shape can make it more difficult to establish long range order in the films.

Previous research has shown that $\pi-\pi$ intermolecular interactions have a strong impact on the conductivity of organic films ${ }^{12-15,19}$. Theoretical calculations also indicate that a well-ordered structure should decrease the tunneling resistance across molecules by the better spatial overlap of the HOMO and LUMO molecular orbitals ${ }^{15}$. Experimental results from Tokumoto et al. ${ }^{13}$ suggest that when load is applied to a well ordered $\pi$-conjugated film of $(1,1 ': 4$ ',1'-terphenyl)-4methanethiol (TPMT) molecules, the AFM tip pushes or penetrates into the film, changing its molecular ordering and decreasing its electrical conductance.

The higher friction measured on the MPAA film could be associated with its more disordered nature because defects can accommodate soft, low-frequency vibrational modes and molecular distortions that favor energy dissipation and result in higher friction force. ${ }^{18,29}$

While molecular order and defect density affect friction, the electrical conductivity is much more critically dependent on the nature of modifications occurring in the films.

In an isolated molecule in the same geometry as in the film, the charge carriers injected from the tip to the $\pi$-conjugated phenyl and anthracene rings must tunnel through the insulating mercaptophenyl segment of the molecule to the gold surface. On a well ordered film with good overlap between the $\pi$-conjugated groups however, the carriers can hope across molecules and thus increase their tunneling probability to the gold due to the many molecules involved.

One can thus envision three very different mechanisms by which conductance can change as a function of load. One is the increase of contact area due to the elastic deformation of tip and surface. This should produce an increase of conductance according to the predictions of contact mechanics, for example as $\mathrm{L}^{2 / 3}$. Another mechanism is a decrease of tip-gold distance due to compression of the film, with possible displacement of molecules at sufficiently high loads. Displacement can result in an ohmic contact of tip with the gold surface. The compression of molecular films as a result of molecular tilts and gauche defect formation induced by the load, has been well documented for alkane chains ${ }^{17}$. Since electron transport through the film between tip and surface is dominated by tunneling, the decrease in tip-surface distance should change the conductance in a much more rapid way than the increase in contact area. Finally, another mechanism is the perturbation of orbital overlap between HOMO and LUMO frontier orbitals due to molecular distortions, which affect particularly the $\pi-\pi$ connection between molecules. Since the topographical height of the MPPA film $(1.0 \mathrm{~nm})$ is smaller than that of MPAA $(1.5 \mathrm{~nm})$, the 10-times higher current measured on MPAA cannot be caused by changes in tip-gold distance, but 
must mainly be the result of intermolecular electrical transport facilitated by favorable $\pi-\pi$ stacking.

On MPPA films, where the molecules have a similar structure as TPMT, conductance increases exponentially with applied load with no abrupt change observed. This suggests that the decrease in the tip-surface distance, and to a much lesser degree the increase in the contact area, dominate the change in conductance in that case.

On the MPAA films the behavior is similar to that of MPPA at low total loads $(0-20 \mathrm{nN})$, so that the current increases due to a decrease in tunneling distance by compression of the film. At high total loads ( $>65 \mathrm{nN}$ ) the increase of current is still nearly exponential and thus again likely to be due to a decrease of the tunneling distance by compression. The rate of increase however is smaller than at low loads reflecting a stiffer less compressive film. The notorious difference with MPPA is the plateau in current between 20-65 nN, which can be regarded as a "decrease" on top of the steady increase caused by the decreasing tunneling distance and increasing contact area. This could be caused by a disruption of the $\pi-\pi$ intermolecular stacking. The lack of plateau in MPPA could be due to a weaker $\pi-\pi$ intermolecular interaction than in MPAA, due to the different sizes and overlaps of the benzene-containing end groups.

A recent review by A. Salomon et. al ${ }^{30}$ suggested that the efficiency of charge transport in $\pi$-conjugated molecules is strongly affected by the HOMO-LUMO gap. However, electron hopping between molecules is also important. Both factors are also strongly affected by the molecular conformation. Since $\pi-\pi$ intermolecular interactions can strongly affect molecular conformation it will also influence the intra molecular HOMO-LUMO gap and therefore the intermolecular charge hopping rate.

\section{Conclusion}

We have compared the molecular conductance change as a function of applied load of two similar $\pi$-conjugated molecules. MPAA, with a higher degree of $\pi$-conjugation, shows a conductivity which is 10 higher than that of MPPA. This indicates that lateral charge transport facilitated by the more efficient $\pi-\pi$ stacking in MPAA plays and important role in the electrical transport properties of conjugated molecules. A plateau in conductance was observed for MPAA as a function of load that could indicate a disruption of the $\pi$ - $\pi$ intermolecular overlap by compressive loads. For MPPA molecule, with a lower degree of $\pi$-conjugation, the conductance increases monotonously with load. 
We found that although the $\pi-\pi$ intermolecular interactions play a relatively less important role in determining the frictional properties of the films, which behave closely as predicted by continuum mechanic models and is therefore most likely dominated by the density of defects in the films.

\section{Acknowledgements}

This work was supported by the Director, Office of Energy Research, Office of Basic

Energy Sciences, Molecular Foundry, Materials Sciences Division, of the U.S. Department of Energy through the Lawrence Berkeley National Laboratory, Contract No. DE-AC02$05 \mathrm{CH} 11231$. 


\section{Reference}

(1) Bao, Z. N. Nature Materials 2004, 3, 137-138.

(2) Burrows, P. E.; Forrest, S. R.; Thompson, M. E. Current Opinion in Solid State \& Materials Science 1997, 2, 236-243.

(3) de Boer, B.; Frank, M. M.; Chabal, Y. J.; Jiang, W. R.; Garfunkel, E.; Bao, Z. Langmuir 2004, 20, 1539-1542.

(4) Dimitrakopoulos, C. D.; Mascaro, D. J. Ibm Journal of Research and Development 2001, 45, 11-27.

(5) Dimitrakopoulos, C. D.; Purushothaman, S.; Kymissis, J.; Callegari, A.; Shaw, J. M. Science 1999, 283, 822-824.

(6) Dodabalapur, A.; Torsi, L.; Katz, H. E. Science 1995, 268, 270-271.

(7) Forrest, S. R. Chemical Reviews 1997, 97, 1793-1896.

(8) Forrest, S. R. Ieee Journal of Selected Topics in Quantum Electronics 2000, 6, 1072-1083.

(9) Heringdorf, F.; Reuter, M. C.; Tromp, R. M. Nature 2001, 412, 517-520.

(10) Knipp, D.; Street, R. A.; Volkel, A.; Ho, J. Journal of Applied Physics 2003, 93, 347-355.

(11)Zareie, M. H.; Ma, H.; Reed, B. W.; Jen, A. K. Y.; Sarikaya, M. Nano Letters 2003, 3, 139-142.

(12)Hunter, C. A.; Sanders, J. K. M. Journal of the American Chemical Society 1990, $112,5525-5534$.

(13) Ishida, T.; Mizutani, W.; Aya, Y.; Ogiso, H.; Sasaki, S.; Tokumoto, H. Journal of Physical Chemistry B 2002, 106, 5886-5892.

(14) Ishida, T.; Mizutani, W.; Tokumoto, H.; Choi, N.; Akiba, U.; Fujihira, M. Journal of Vacuum Science \& Technology a-Vacuum Surfaces and Films 2000, 18, 14371442.

(15) Magoga, M.; Joachim, C. Physical Review B 1999, 59, 16011-16021.

(16) Dou, R. F.; Ma, X. C.; Xi, L.; Yip, H. L.; Wong, K. Y.; Lau, W. M.; Jia, J. F.; Xue, Q. K.; Yang, W. S.; Ma, H.; Jen, A. K. Y. Langmuir 2006, 22, 3049-3056.

(17) Barrena, E.; Kopta, S.; Ogletree, D. F.; Charych, D. H.; Salmeron, M. Physical Review Letters 1999, 82, 2880-2883.

(18)Xiao, X. D.; Hu, J.; Charych, D. H.; Salmeron, M. Langmuir 1996, 12, 235-237.

(19) Bhushan, B.; Liu, H. W. Physical Review B 2001, 6324.

(20) Liu, H.; Bhushan, B. Ultramicroscopy 2002, 91, 185-202.

(21) Park, J. Y.; Ogletree, D. F.; Salmeron, M.; Jenks, C. J.; Thiel, P. A. Tribology Letters 2004, 17, 629-636.

(22) Park, J. Y.; Ogletree, D. F.; Salmeron, M.; Ribeiro, R. A.; Canfield, P. C.; Jenks, C. J.; Thiel, P. A. Physical Review B 2005, 71, 144203.

(23) NT-MDT Co., Zelenograd Research Institute of Physical Problems, Moscow, Russia.

(24) Park, J. Y.; Ogletree, D. F.; Thiel, P. A.; Salmeron, M. Science 2006, 313, 186186.

(25) Size of van der Waals structure of MPAA is 1.56nm (height) $x 1.15 \mathrm{~nm}$ (width), and that of MPPA is $1.56 \mathrm{~nm}$ (height) and $0.67 \mathrm{~nm}$ (width). See reference 11. 
(26) The relation between friction force and contact area can be described by the Derjaguin-Muller-Toporov (DMT) or the Johnson-Kendall-Roberts (JKR) models, depending on the adhesion force and hardness of the sample. Generally, the JKR model is valid for large tip radius and large adhesion, while DMT applies best in the case of small radius and low adhesion.

(27) Carpick, R. W.; Agrait, N.; Ogletree, D. F.; Salmeron, M. Journal of Vacuum Science \& Technology B 1996, 14, 1289-1295.

(28) Johnson, K. L.; Kendall, K.; Roberts, A. D. Proceedings of the Royal Society of London Series a-Mathematical and Physical Sciences 1971, 324, 301-\&.

(29) Salmeron, M. Tribology Letters 2001, 10, 69-79.

(30)Salomon, A.; Cahen, D.; Lindsay, S.; Tomfohr, J.; Engelkes, V. B.; Frisbie, C. D. Advanced Materials 2003, 15, 1881-1890. 